\title{
Attraction between DNA molecules mediated by multivalent ions
}

\author{
E. Allahyarov, ${ }^{1}$ G. Gompper, ${ }^{1}$ and H. Löwen ${ }^{2}$ \\ ${ }^{1}$ Institut für Festkörperforschung, Forschungszentrum Jülich, D-52425 Jülich, Germany \\ ${ }^{2}$ Institut für Theoretische Physik II, Heinrich-Heine-Universität Düsseldorf, D-40225 Düsseldorf, Germany
}

(Received 13 November 2003; revised manuscript received 12 January 2004; published 28 April 2004)

\begin{abstract}
The effective force between two parallel DNA molecules is calculated as a function of their mutual separation for different valencies of counterion and salt ions and different salt concentrations. Computer simulations of the primitive model are used and the shape of the DNA molecules is accurately modeled using different geometrical shapes. We find that multivalent ions induce a significant attraction between the DNA molecules whose strength can be tuned by the averaged valency of the ions. The physical origin of the attraction is traced back either to electrostatics or to entropic contributions. For multivalent counterions and monovalent salt ions, we find a salt-enhanced repulsion effect: the force is first attractive but gets repulsive with increasing salt concentration. Furthermore, we show that the multivalent-ion-induced attraction does not necessarily correlate with DNA overcharging.
\end{abstract}

DOI: 10.1103/PhysRevE.69.041904

PACS number(s): 87.15.Kg, 61.20.Ja, 82.70.Dd, 87.10.+e

\section{INTRODUCTION}

During the last decade the question that concerns the possible existence of attractive interactions between similarly charged objects in electrolyte solutions has been intensely debated. Experimental evidence of such an attraction is seen for deoxyribose nucleic acid (DNA) molecules [1-4], colloidal rods [5], charged clay particles [6], charged microspheres [7-9], and charged plates $[10,11]$. In particular, DNA molecules in solution are a paradigm for negatively charged polyelectrolytes due to ionization of its acidic phosphate groups [12]. The DNA conformations display a considerable sensitivity to the ionic surrounding. The mutual repulsion of DNA polyions has to be overcome to form compact or condensed DNA bundles. Experiments show that DNA condensation occurs when about $90 \%$ of its charge is neutralized by condensed counterions $[1,2,13,14]$. Such a strong neutralization of the DNA charge could be achieved by divalent and higher-valent counterions $[15,16]$. Besides the phosphate neutralization, the multivalent ions induce an additional attraction between the DNA macroions mediated by strong correlation effects [14,17-24]. Thus the small ions play a complex role in DNA-DNA interactions and are not simply agents to screen the long-range electrostatic interaction. For example, they adsorb onto the DNA surface and can create bridges between the DNA molecules at small DNA-DNA separations, resulting in an ion cross-link attraction [22,25].

The electrostatic interaction between highly charged polyelectrolytes is usually treated within the framework of classical double-layer theory [26]. This theory is based on the mean-field Poisson-Boltzmann equation [27-34] and predicts a repulsion between similarly charged macromolecules. Though different modifications of Poisson-Boltzmann theory have been developed to account for ion-ion hard core correlations [35-38], an attractive contribution in the double-layer theory is usually introduced via the van der Waals interaction forces [7,39-41]. However, the van der Waals forces alone cannot explain the experimentally observed attraction, since the Hamaker constant extracted from the experiments is artificially high [7,42-44].
Theoretical investigations and numerical simulations indicate that an attraction between similarly charged objects emerges beyond the mean-field approaches. It is now a well established fact that the charge correlations and fluctuations in highly charged electrolytes can induce an attraction between the macroions [21,45-67]. Due to the very resemblance of the short DNA fragments to charged rods, the latter is a widely used toy model for the DNA molecule in theoretical treatments and computer simulations $[22,28,61,68-73]$. However, the details of DNA, such as the discreteness and helical structure of the DNA phosphate charges and the grooved shape of the DNA molecule, become essential as one approaches its surface. In this case, strictly speaking, all atom DNA simulations in molecular water would be a proper choice [74]. Unfortunately such sophisticated simulations can only be applied to small systems and small salt concentrations [75]. Thus, first, a design of a "sophisticated" DNA model, which goes beyond the simple homogeneously charged cylinder model, and second, an investigation of the interaction forces between such DNA molecules, remains a challenging task.

This paper is an extension of our previous works on DNA electrostatics [76,77]. In Ref. [76], simulation results for the DNA-DNA interaction were compared with the predictions of different linear theories for the case of monovalent ions. It was shown that the DNA-DNA interaction, at separation distances smaller than the Debye screening length, differs from the predictions of mean-field theories. This provides evidence that the intermolecular interaction depends not only on how many ions are in the DNA proximity (which is exactly what the ordinary linear theories rely on), but also on where those ions are located relative to the DNA structure, i.e., whether they penetrate into the grooves or not. In Ref. [77], on the other hand, a detailed distribution of ions of different valencies and molarities near the DNA surface was explored for a more realistic, grooved shape of the DNA molecule. The results obtained indicate that the paths of counterion and coion condensations strongly depend on the DNA surface geometry. Taking this into account we expect that the implemented DNA models with different geometries will also af- 
fect significantly the effective DNA-DNA interaction. Thus, in this paper, we will focus on the mechanism of attraction between two DNA molecules shaped similar to models introduced in Ref. [77]. Our goal is to see the effects, which increasing detail of various DNA models have on the DNADNA interaction. We show that the DNA shape is an essential contributor to the interaction force for multivalent counterions, whereas it has a minor effect on the interaction force for added multivalent salt. The origin of the attraction in the simple and sophisticated DNA models is different. For instance, a Coulomb depletionlike attraction [78] for the saltfree case depends on the implemented DNA model. It has been revealed that there is a nonmonotonic force-salt dependence at a fixed DNA-DNA separation for added monovalent salt and divalent counterions. This is exemplified by the variation of the interaction force from a strong attraction towards a strong repulsion and a following decrease in magnitude. Detailed investigations connect this "salt-enhanced repulsion" to the entropic part of the total interaction force. We also address the competition between the multivalent counterion and the multivalent salt-induced attractions. It is shown that the increase of the divalent salt concentration at a fixed monovalent ion number drives the DNA-DNA interaction force into an attraction through the overcharging of DNA molecules. However, the DNA-DNA attraction induced by trivalent counterions decreases, while the DNA molecule gradually gets overcharged due to added divalent salt.

The remainder of the paper is organized as follows. We give a short general overview of ion binding and DNA condensation in Sec. II. The system parameters and quantities studied in the present work are discussed in Sec. III. Sections IV and $\mathrm{V}$ contain simulation details and the implemented simulation techniques. The specific DNA configurations at short DNA-DNA separations are discussed in Sec. VI. Sections VII and VIII are devoted to simulation results for monovalent and divalent salt ions, respectively. We conclude in Sec. IX.

\section{ION BINDING AND DNA CONDENSATION}

There are essentially two contenders for the dominant attractive force in the DNA condensation: hydration forces [79] and correlated counterion charge fluctuations. Throughout this paper we neglect the granular nature of water and the solvent-induced forces, and concentrate only on the electrostatics of the DNA condensation. The water dielectric effects and hydration forces will be briefly (and qualitatively) discussed in Sec. IX.

Under physiological conditions, the DNA molecule is surrounded by an ionic atmosphere with a Debye screening length $\lambda_{D}$ in the range of $5-10 \AA$. Within the distances $r<\lambda_{D}$ above the DNA surface, a nonlinear screening of the DNA phosphate charges takes place. Hence, if the surfaceto-surface separation between two DNA molecules is less than $\lambda_{D}$, a nonlinear theory [80] has to be applied. At surface-to-surface separation distances on the order of or beyond $\lambda_{D}$, Debye-Hückel theory (based on a linearized Poisson-Boltzmann treatment) is a reasonable approximation to describe the ionic atmosphere around the DNA molecule.
In Ref. [76] we have examined several mean-field theories for their ability to match the numerically calculated DNADNA interaction forces: the homogeneously charged rod model, the Yukawa segment (YS) model, and the KornyshevLeikin (KL) theory [81]. For the case of an overall monovalency of counterions and salt ions, both the simulations [76] and the above mentioned theories reveal repulsive forces between the DNA molecules for all mutual orientations and separation distances. We have shown that, except for short separation distances, there is a qualitative agreement between the theoretical and numerical results if a proper charge and size renormalization in the former is performed.

For multivalent counterions and added salt ions, there is experimental evidence that the DNA molecules attract each other. Such an attraction is completely missed in the linear theories such as the homogeneously charged cylinder and YS model. In these theories all the nonlinear salt effects are again accounted for through the phosphate charge and screening length renormalization procedure. Only the meanfield KL theory predicts a DNA-DNA attraction for some DNA-DNA separations and azimuthal molecular orientations. In detail, the KL theory distinguishes between strongly condensed (also called as bound or adsorbed) and a cloud of diffusive (nonbonded) counterions. A tight adsorption is assumed to take place within the Stern layer of thickness $\xi$ $=A / 4 \pi \lambda_{B}=2 \AA$, where $\lambda_{B}=e^{2} /\left(\epsilon k_{B} T\right)$ is the Bjerrum length, $A$ is an average area per elementary charge on the DNA surface, $\epsilon$ is a dielectric constant of solution, and $k_{B} T$ is the thermal energy. The KL theory [81] predicts an attractive force between the two DNA molecules if the following conditions are fulfilled: (i) more in-groove than on-strand condensation, (ii) the right complementary alignment of the positively charged grooves on one helix facing the negatively charged strand on the other helix. In other words, the KL theory assumes that it is the DNA charge helicity that entails an intermolecular attraction for surface-to-surface distances in the range of $8-15 \AA$. Theoretical results and computer simulations [33,76,82-84], however, indicate that no chargehelicity effects extend further than few angstrom from the DNA surface. There is also experimental evidence [85] that at surface-to-surface separation distances comparable with the Debye screening length, the DNA-DNA separation does not affect the DNA orientation.

The discreteness of the DNA phosphates, explicitly taken into account by our DNA models, enhances the counterion concentration and the surface adsorption of ions [86] through the increased Coulomb coupling between the phosphates and the counterions. This boosts the counterion correlations near the DNA surface. Experiments indicate that the divalent counterions, depending on their in-groove or on-strand localization, have different impact on the DNA systems. Thus, the transition metals with higher affinity to the DNA bases condense on DNA, while alkali metals do not [2]. On the other hand, the chemical identity of the cation is a factor of minor importance compared with the magnitude of their charge when $q_{c}>2$ [2].

\section{SYSTEM PARAMETERS}

\section{A. DNA models}

The $\mathrm{B}$ form of DNA has an inner core of radius $9 \AA$ formed by nucleotide pairs, and two sugar-phosphate strands 
spiralling around it. The latter form the well-known double helix with a pitch length $P$ of about $34 \AA$ [12]. There are two phosphate groups per base pair, and 10 base pairs per pitch length, which makes $N_{p}=20$ phosphate charges per one helical turn. The axial rise per base pair in the DNA long axis is $3.4 \AA$, thus there is one elementary charge per $1.7 \AA$. The average value of the angle between the adjacent base pairs is $36^{\circ}$ and the average distance between the neighboring charges on the DNA surface is about $7 \AA$. This distance is much smaller than the helical pitch and of the order of Debye screening length under the physiological conditions. There is a small shift in the $z$ coordinate of two opposing phosphates belonging to different helices of DNA, $\delta z=0.34 \AA$.

Three DNA models, a cylinder model (CM), an extended cylinder model (ECM), and the Montoro-Abascal model (MAM), are considered. Our aim is to obtain a detailed understanding of the physical mechanism of ion-mediated DNA interactions, in particular how the geometry of different DNA models gives rise to new effects. The CM has a hard cylindrical core of diameter $D=20 \AA$ and two strings of monovalent phosphates of size $d_{p}=0.4 \AA$. The KL theory, and almost all the Poisson-Boltzmann like theories and most of primitive model (PM) computer simulations, have utilized the CM as a simple DNA model. In the ECM, first designed by Lyubartsev et al. [82], the helical grooves of DNA are incorporated through the shrinking of the DNA core to the size $D=17.8 \AA$ and swelling the phosphate spheres to the size $d_{p}=4.2 \AA$. A grooved structure, which resembles the real DNA appearance, is achieved in the MAM [83] through adding another neutral sphere between the cylindrical core and the charged phosphate sphere. The cylindrical core in the MAM has a diameter $D=7.8 \AA$, the inner string of neutral spheres is centered at a radial distance $r=5.9 \AA$, and the outer string of phosphates is centered at a radial distance $r$ $=8.9 \AA$. Both spheres have the same $\phi$ and $z$ coordinates and diameter $d_{p}=4.2 \AA$. A full description of these models is given in Refs. [76,77,83].

In addition to the two DNA molecules the system contains counterions of charge $q_{c}$, symmetric salt ions of concentration $C_{s}$, and charges $q_{+}$and $q_{-}$. All the small ions are modeled as hard spheres of a diameter $d_{c}$ for counterions, $d_{+}$and $d_{-}$for the salt ions. The whole system is held at room temperature $T=298 \mathrm{~K}$. The primitive model simulations with no explicit water deal only with a passive (nonspecific) binding and completely neglect the specific binding of counterions to the DNA grooves. In this case the ion binding sites are determined by the steric and Coulombic interactions [77].

The interactions between the mobile ions and the phosphate charges are described within the framework of primitive model as a combination of the excluded volume and Coulomb interactions reduced by the inverse of the dielectric constant $\epsilon$ of the solvent. The corresponding pair interaction potential between the different charged hard spheres is

$$
V_{i j}(R)=\left\{\begin{array}{l}
\infty \quad \text { for } r \leqslant\left(d_{i}+d_{j}\right) / 2 \\
\frac{q_{i} q_{j} e^{2}}{\epsilon R} \quad \text { for } R>\left(d_{i}+d_{j}\right) / 2,
\end{array}\right.
$$

where $R$ is an interparticle separation distance, $i$ and $j$ are indices denoting the different particles species. Possible val- ues for $i$ and $j$ are $c$ (for counterions),,+- (for positively and negatively charged salt ions), $p$ (for phosphate groups), and $n$ (for the neutral spheres in the MAM with $q_{n}=0$ ). In addition, there is an interaction potential $V_{i}^{0}$ between the DNA hard cylinder and the free ions $i=c,+,-$. This potential has a simple excluded volume form such that the free ions cannot penetrate into the cylinder.

\section{B. Simulated quantities}

Our basic simulated quantity is the effective force $[76,78]$ between the DNA molecules

$$
\vec{F}=\vec{F}_{1}+\vec{F}_{2}+\vec{F}_{3} .
$$

Here $\vec{F}_{1}$ is the direct Coulomb force acting onto all the phosphate charges belonging to one helical turn of one DNA molecule as exerted from the phosphate groups of the other DNA,

$$
\vec{F}_{1}=-\sum_{k}{ }^{\prime}\left(\vec{\nabla}_{r_{k}^{p}} \sum_{n=1}^{N_{p}} V_{p p}\left(\left|\vec{r}_{k}^{p}-\vec{r}_{n}^{p}\right|\right)\right) .
$$

The sum $\Sigma_{k}^{\prime}$ only runs over phosphates of one helical turn of the DNA molecule.

The second term $\vec{F}_{2}$ corresponds to the Coulomb interactions between the phosphate charges and the mobile salt ions. This term describes the screening of the DNA charge,

$$
\vec{F}_{2}=-\sum_{k}{ }^{\prime}\left(\left\langle\sum_{i=c,+,-} \sum_{l=1}^{N_{i}} \vec{\nabla}_{\vec{r}_{k}^{p}} V_{p i}\left(\left|\vec{r}_{k}^{p}-\vec{r}_{l}^{i}\right|\right)\right\rangle\right) \text {. }
$$

The third term $\vec{F}_{3}$ arises from the entropic contribution of small ions due to their excluded volume interaction with the DNA molecular surface $\mathcal{S}_{i}$. Its value for one helical turn is

$$
\vec{F}_{3}=-k_{B} T \int_{\mathcal{S}_{i}} d \vec{f}\left(\sum_{j=c,+,-} \rho_{j}(\vec{r})\right)
$$

where $\vec{f}$ is a surface normal vector pointing outwards the DNA core. This term becomes increasingly important as the Coulomb coupling parameter $\Gamma_{p c}$ is elevated for the multivalent counterions [76,78],

$$
\Gamma_{p c}=\left|\frac{q_{p}}{q_{c}}\right| \frac{2 \lambda_{B}}{d_{p}+d_{c}} .
$$

The parameter $\Gamma_{p c}$ determines the importance of thermal fluctuations. When $\Gamma_{p c}>1$, the Coulomb interaction energy between the DNA and the surrounding salt ions dominates over the thermal fluctuations in system.

\section{COMPUTER SIMULATIONS}

We consider two parallel DNA molecules, separated by a distance $R$ along the $x y$ diagonal of the cubic simulation box of size $L$ and volume $V=L^{3}$. The size of the simulation box $L=102 \AA$ corresponds to the three full turns of B-DNA [83]. The box also consists $N_{c}$ counterions and $N_{+}=N_{-}=N_{s}$ salt ions of both signs. The counterion concentration is fixed by 


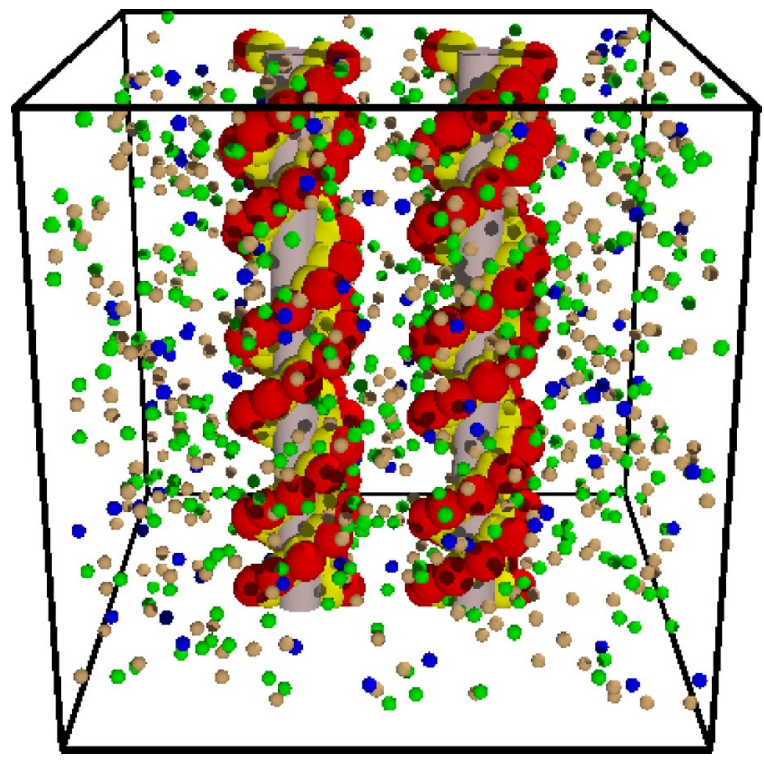

FIG. 1. (Color online) A typical snapshot of the simulation box. The DNA molecules are drawn according to the MAM. Black spheres on the DNA strands represent the phosphate charges. Internal gray spheres between the phosphates and the DNA cylindrical core are neutral. Positive (negative) salt ions spread across the simulation volume are shown as open (hatched) spheres.

the charge of DNA molecules in the simulation box due to the constraint of global charge neutrality. A typical snapshot of the simulation cell is illustrated in Fig. 1. Periodic boundary conditions in all three directions are applied to reduce the confined volume effects in electrolytes. The DNA replicas in the $z$ direction produce an infinitely long DNA molecule which avoids the end effects encountered in other molecular simulations of short DNA segments [87]. The phosphate spheres are monovalent, $q_{p}=-e$, where $e$ is an elementary charge. The dielectric permeability $\epsilon$ is considered to be a constant everywhere in system, which avoids the need of electrostatic images. The long-range interactions between the two charged species and their replicas in the neighboring cells are handled via the Lekner method [88] and its modification for the particular cases, when pairs of interacting charges are sitting exactly on one of the coordinate axes (this case was elaborated in the Appendix of Ref. [76]).

We have performed extensive molecular dynamics (MD) simulations for a range of different microion valencies. The simulated states are given in Table I. The ion diameter was chosen to be $d_{c}=3 \AA$. This parameter defines the closest ap-

TABLE I. Parameter sets used for the simulations of DNA-DNA interactions.

\begin{tabular}{ccc}
\hline \hline Set & $q_{c}$ & $q_{s}$ \\
\hline 1 & 1 & 1 \\
2 & 2 & 1 \\
3 & 3 & 1 \\
4 & 1 & 2 \\
5 & 3 & 2 \\
\hline \hline
\end{tabular}

proach of the ion to the DNA surface and has a strong impact on the polyion electrostatics $[18,89]$. A test simulation for an increased ion diameter $d_{c}=5 \AA$, which mimics the ion hydration in solvent $[18,77,89]$, shows no qualitative changes of the reported results. We treat the ions as condensed if the surface-to-surface distance between the ion sphere and the DNA hard surface is not larger than $2 \AA$.

During the simulations, we calculate the interaction forces between the two DNA molecules for different separation distances $R$. Due to the strong screening of the DNA phosphates, the actual salt concentration in the bulk of the simulation box $C_{s}^{\prime}(R)$, measured far away from the polyelectrolytes, is $R$ dependent and is always smaller than the nominal salt concentration, $C_{s}^{\prime}(R)<C_{s}=N_{s} / V$. Thus, an implementation of the conventional MD procedure with a fixed ion number $N_{s}$ gives interaction forces which correspond to different bulk densities $C_{s}^{\prime}(R)$ for each intermolecular distance $R$. This problem can be avoided by considering a solution with a constant chemical potential $\mu$ via the grand canonical (GC) simulation method [65]. The GC simulation is a natural choice to mimic the experimental situation where the actual salt concentration of the ordered DNA phase is not known a priori. Instead, it is given by the thermodynamic condition that the chemical potential $\mu$ in the DNA solution has to be the same as in the bulk electrolyte phase with which it is in equilibrium and whose concentration $C_{s}$ is experimentally known. Thus the number of ions in the simulation cell is automatically adjusted to the specified value of chemical potential $\mu$, which, in turn, is linked to the concentration of ions in the bulk phase $C_{s}$ [90]. In the present paper a combination of different grand canonical molecular dynamics (GCMD) schemes is used which is optimally suited for our task.

\section{GRAND CANONICAL MOLECULAR DYNAMICS}

In addition to the usual propagation of the particles, the conventional GC simulation technique [90] consists of the creation of particle at a random position in the simulation box or destruction of a randomly chosen particle. Each of these moves is associated with a probability of acceptance, which is determined by the ratio of two Boltzmann factors. In application to electrolytes a modified GC method was devised in Refs. [69,91], where the insertion or removal of a pair of ions of the same valency and opposite charge is done simultaneously to keep the system electroneutral. Unfortunately, these moves have relatively low acceptance rates for dense and multivalent salt solutions [69], making the simulations inefficient.

Another challenge in the GC simulations is the apparent incompatibility of the deterministic and stochastic approaches. The dynamical information will be adversely affected when particles suddenly appear and disappear. This effect becomes even more pronounced for a nonhomogeneous system [53,54], like a DNA immersed in solution, where an artificial and unrealistic ion flow toward the DNA surface appears. This will further destabilize the system equilibrium. To minimize this inconsistency of the system dynamics, a method of local potential control (LPC), first in- 
troduced in Ref. [92], can be adopted. Within the LPC method, the creation and destruction of particles is restricted to a control volume. The other possibility is a procedure developed by Attard in Ref. [93], where the GCMD is performed with a fixed number of particles by coupling the variations in the system size to the instantaneous chemical potential determined by the virtual test particle method. This method also cures the low acceptance rates of particle insertions and deletions for dense systems. In the present simulations we take advantage of both the above mentioned methods [92,93]. In detail, we first determine the specified nominal chemical potential $\mu$ of the bulk electrolyte in the absence of DNA molecules via a modified Widom method with multiparticle insertion [94]. Then we match the actual chemical potential $\mu^{\prime}$ to the nominal chemical potential $\mu$ using a GCMD simulation similar to the method invoked in Ref. [92] and locate the control volume near the cell boundary. At each time step an equal but arbitrary number of creation/deletion attempts are made in the control volume. After a successful creation, a velocity is drawn from the Maxwell-Boltzmann distribution at a temperature $T$ and assigned to the new particle. In the first stage the particle number $N_{s}^{\prime}$ in the simulation box increases monotonically from its initial value $N_{s}$ given in Table I. Then $N_{s}^{\prime}$ approaches its saturated value and starts to fluctuate around it. This is followed by the fluctuation of instantaneous chemical potential $\mu^{\prime}$ around the $\mu$. At this stage we fix the particle number and allow the system size to fluctuate according to the procedure given by Attard in Ref. [93]. The fluctuations along the $x$ and $y$ directions ( $z$ direction is strictly bound to the DNA length) never exceed a few percents of the box size $L$. Our test simulations with and without the Attard method [93] show the equivalence of the algorithms, with the former being much faster.

\section{MUTUAL DNA CONFIGURATIONS}

We calculate the total interaction force $F(R)$, Eq. (2), and its components $F_{2}(R)$ and $F_{3}(R)$, compare Eqs. (4) and (5), respectively, for a given nominal salt concentration $C_{s}$. The direct phosphate-phosphate interaction $F_{1}(R)$, Eq. (3), does not depend on the salt density and its assessment is straightforward. It should be mentioned that in addition to the separation distance $R$, there are three angular variables which define the mutual configuration of two DNA molecules. These variables, the azimuthal angles $\phi_{s}, \phi_{o}$, and $\phi$ are shown in Fig. 2. The angle $\phi_{s}$ defines the widths of the DNA grooves [77] in the $x y$ plane, it is $134^{\circ}$ for the CM and ECM, and $154^{\circ}$ for the MAM. The parameter $\phi_{0}$ is the angle between the phosphate charge and the DNA-DNA separation vector $\vec{R}=\vec{R}_{1}-\vec{R}_{2}$ and characterizes the discrete location of the phosphate charges along the strands. All the results for the DNA-DNA interaction are periodic in $\phi_{0}$ with a periodicity of $36^{\circ}$. The angle $\phi$ describes the rotation of the second DNA cylinder around its long axis with regard to the first DNA cylinder. There are five particular DNA-DNA configurations which make a strong contribution to the interaction force at short separation distances [76]. Three of these configurations correspond to the case when the phosphate

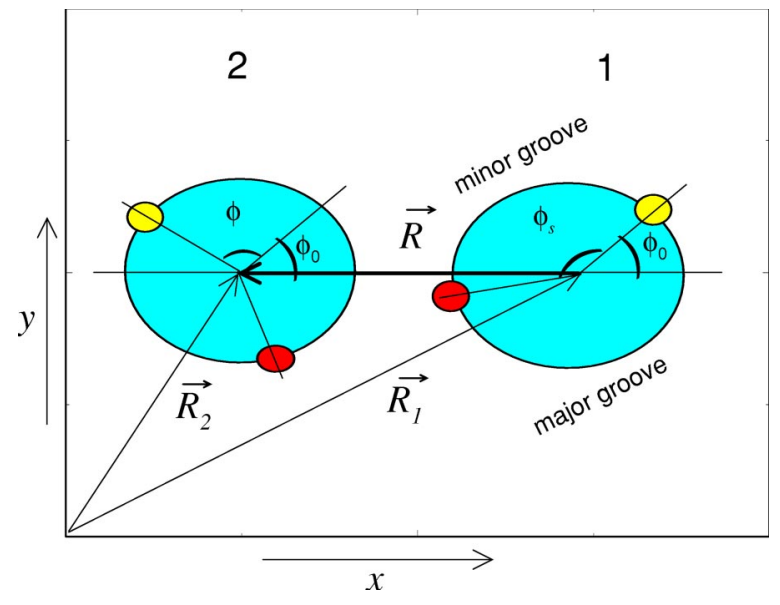

FIG. 2. (Color online) A schematic picture explaining the positions of DNA molecules and the definition of the different azimuthal angles $\phi_{s}, \phi_{0}, \phi$. For further information, see text.

charges of neighboring DNA molecules are "touching," see Fig. 3. The other two particular configurations correspond to the so called "DNA zipper" situation, when the strands of one DNA stand against the grooves of the neighboring DNA. This happens when $\phi= \pm 3 \pi / 5$ regardless the value of $\phi_{0}$. Our previous [76] and present simulations prove that the interaction force does depend on the mutual DNA configurations at a short separation distances $R<25 \AA$, or when the surface-to-surface distance between the two DNA molecules is less than $5 \AA$. This follows mainly from ion bridging between the two neighboring phosphates via a positive salt ion in configurations pictured in Fig. 3, or from sharing an adsorbed salt ion in one DNA groove and on the other DNA strand in the DNA zipper configurations. In fact, for such small separations, comparable with the solvent particle size, discrete solvent effects will show up in vitro. On the other hand, the multivalent ions increase the hydrodynamic radius of the DNA molecule, which in turn makes it unlikely for two neighboring DNA molecules to come closer than the contact shell-to-shell distance [89]. Arguments against the existence of cross links for multivalent ions are given in Ref. [1] in order to explain the fluidity of the condensed phase of DNA system. Thus, numerical results for small DNA separations, accessible in simulations but subject to a complicated statistical averaging procedure, bear no physical meaning to match the experimental results. For larger separation distance, $R>25 \AA$, we find no detectable dependence of the interaction forces on the azimuthal angles $\phi_{0}$ and $\phi$. This is in accordance with the early reports $[33,82-84]$ that the helicity and discreteness effects of the DNA charges are generally small and dwindle a few angstroms away from the DNA surface. In all figures hereafter we show the interaction forces starting from the distances $R=24 \AA$ and for azimuthal angles $\phi_{0}=18^{\circ}$ and $\phi=0^{\circ}$. The interaction forces are scaled per DNA pitch, i.e., per 10 DNA base pairs. A positive sign of the forces denotes a repulsion, while a negative sign denotes an attraction. The cases of monovalent and multivalent salt ions are considered separately. 

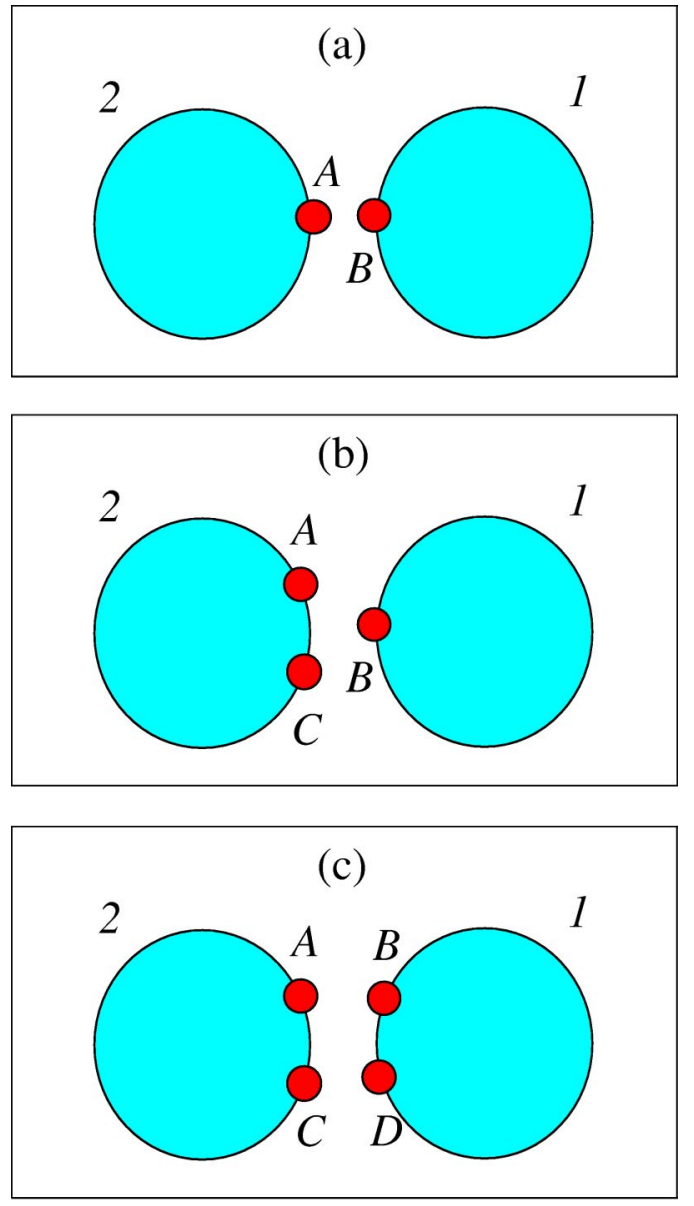

FIG. 3. (Color online) Three typical configurations for the two parallel DNA molecules when their phosphates charges are close to each other. The $x y$-plane cross sections of DNA molecules are shown for the ECM. Note that only the neighboring phosphates in the inter-DNA area are shown, see the dark small circles labeled by letters $A, B, C, D$. (a) $\phi_{0}+n \phi_{s}=\pi, \phi=\pi, \pi / 5$. (b) $\phi_{0}+n \phi_{s}=\pi, \phi$ $=\pi-\phi_{s} / 2, \pi / 5-\phi_{s} / 2$. (c) $\phi_{0}+n \phi_{s}=\pi \pm \phi_{s} / 2, \phi=\pi, \pi / 5$. Here $n$ is an integer number, $n=0, \pm 1, \pm 2, \ldots$ In (b) and (c) the pair of phosphates on each cylinder pertain to the same strand and have different $z$ coordinates: (b) $z_{A}=z_{B}-(1.7 \AA), z_{c}=z_{B}+(1.7 \AA)$; (c) $z_{A}=z_{D}$, $z_{B}=z_{C}, z_{A}-z_{C}=3.4 \AA$.

\section{SIMULATION RESULTS FOR MONOVALENT SALT}

\section{A. Monovalent counterions}

The calculated DNA-DNA interaction forces for monovalent salt and counterions are depicted in Fig. 4. All three DNA models exhibit a repulsion between the DNA molecules for all calculated separation distances and salt densities shown in Fig. 4 [2,76]. The repulsion in the CM is roughly twice as strong as in the MAM. This is a result of grooved nature of the MAM where the vast majority of adsorbed counterions sits in the minor groove [77]. At large separation distances there is an influence of neighboring DNA molecules on the interaction forces due to the periodically repeated simulation box. This effect is of the order of $5-10 \%$ at $R=40 \AA$ for salt densities less than $0.1 \mathrm{~mole} / 1$ in Fig. 4. The corresponding screening lengths are indicated in

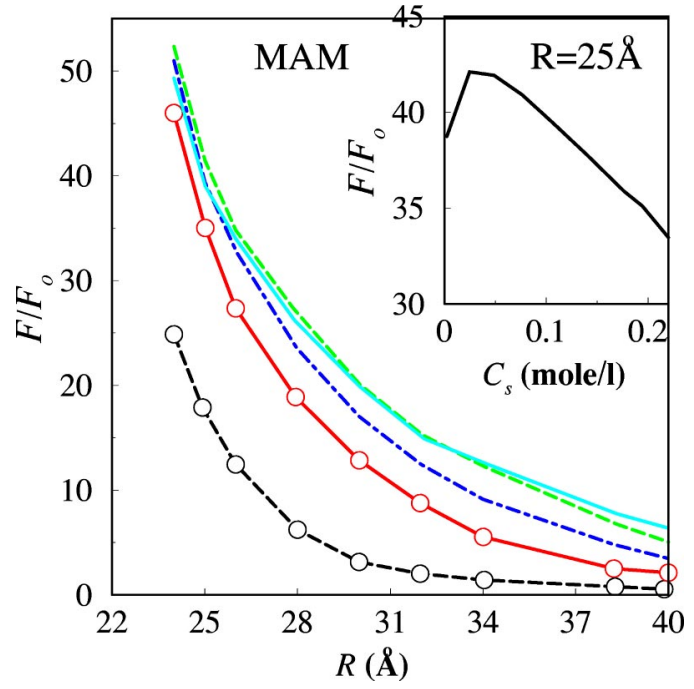

FIG. 4. (Color online) Reduced DNA-DNA interaction force $F / F_{0}$ vs separation distance $R$ for the monovalent counterions and monovalent salt ions (parameter set 1 of Table I) for the MAM. The $\mathrm{CM}$ and ECM exhibit similar trends. The unit of the force is $F_{0}$ $=k_{B} T / P$, where $P$ is the DNA pitch length. Different salt densities are shown: $C_{s}=0 \mathrm{~mole} / 1$ (solid line), $0.024 \mathrm{~mole} / 1$ (dashed line), 0.097 mole/1 (dot-dashed line), 0.194 mole/1 (solid line with symbols), 0.71 mole $/ 1$ (dashed line with symbols). The corresponding screening lengths are $\lambda_{D}=9.6 \AA, 9 \AA, 8 \AA, 5.6 \AA, 3.3 \AA$. The inset shows the force-salt nonmonotonicity at the separation distance $R$ $=25 \AA$.

the caption of Fig. 4. For multivalent ions considered in the following sections with smaller screening lengths, the effect of images is smaller and can be completely neglected.

The salt dependence of the force at a fixed, small separation appears to be nonmonotonic. In detail, if the salt molarity is increased from $C_{s}=0 \mathrm{~mole} / 1$ to $C_{s}=0.024 \mathrm{~mole} / 1$, the repulsion at short distances becomes stronger, see the inset of Fig. 4. Though this trend is at odds with the classical screening theories, a similar effect has already been reported in Ref. [76]. A detailed consideration of the interaction force components (not shown here) reveals that the nonmonotonicity of the interaction force $F(R)$ has a purely electrostatic origin. The nonmonotonicity aggravates for the divalent counterions and induces a switch from attraction to repulsion, see the next subsection.

\section{B. Divalent counterions}

The interaction forces for divalent counterions and monovalent salt, given in Fig. 5, reveal a DNA-DNA attraction for small added salt concentrations. First we analyze the salt free case, when the attraction in the CM is nearly three times stronger than in the MAM. In the cylindrical model the attraction is totally associated with the "Coulomb depletion" effect [78], which is related to the formation of strongly correlated counterion liquid on the DNA surface. For short DNA-DNA separations, when the mean separation distance between counterions on the DNA surface exceeds the separation distance $R$, the two strongly correlated counterion clouds on different DNA rods repel each other. This leads to 

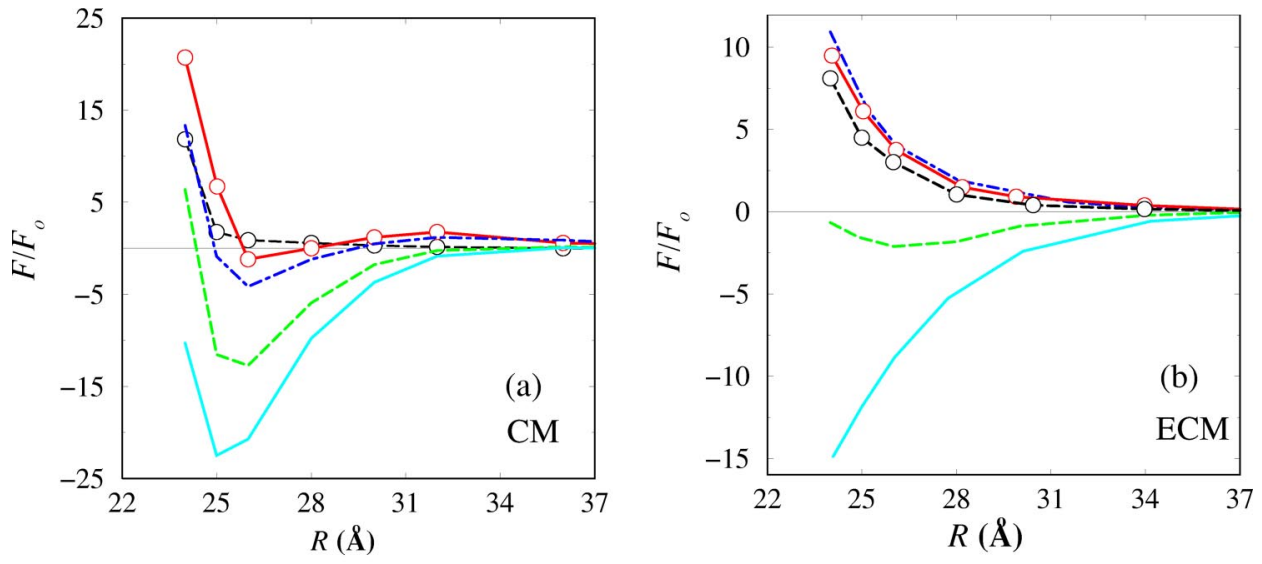

FIG. 5. (Color online) Reduced DNA-DNA interaction force $F / F_{0}$ vs separation distance $R$ for divalent counterions and monovalent salt ions (parameter set 2 of Table I). (a) CM, (b)

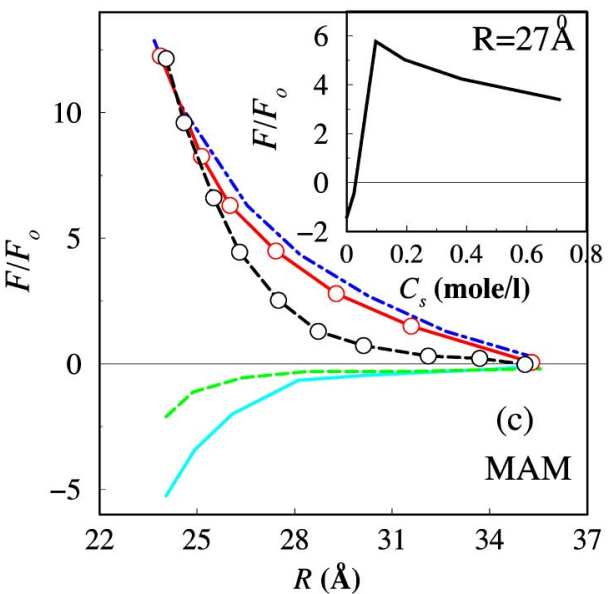
ECM, and (c) MAM. The notation is the same as in Fig. 4. The inset in (c) shows the force-salt nonmonotonicity at the separation distance $R=27 \AA$.

unbalanced and attractive pressure from the outer counterions [95], see solid lines in Fig. 6. It is worth to mention that in Ref. [78] such a correlation mechanism was reported for a spherical colloid with a central charge in a low dielectric medium. Surprisingly, for the cylindrical macroions with a discrete surface charge considered here, we recapture a similar effect.

Contrary to the CM, the attraction in the MAM (and in the ECM as well) has a purely electrostatic origin, as proven by Fig. 7. There are more ions in the inter DNA-DNA area compared to the outer DNA-DNA area. The range and strength of this attraction is higher for the ECM than for the
MAM. To our belief, this is due to the different counterion condensation patterns on the DNA surface in the MAM and ECM. In the latter model the ions predominantly adsorb on the DNA strands and in the minor groove. Therefore they occupy more DNA surface area compared to the MAM, where the main destination of the ion adsorption is the minor groove of DNA [77].

Upon an addition of monovalent salt, the trend in the interaction force depends sensitively on the DNA shape which is modeled differently in the CM, ECM, and MAM. In the CM the DNA-DNA attraction, purely of electrostatic origin, persists to high salt concentrations. Only at short sepa-

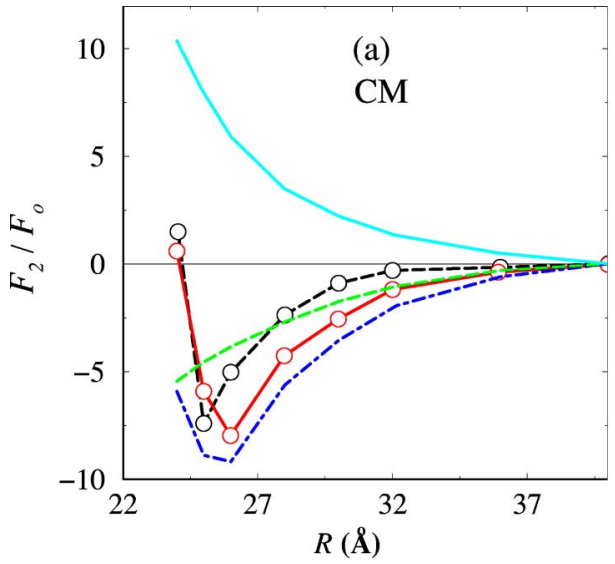

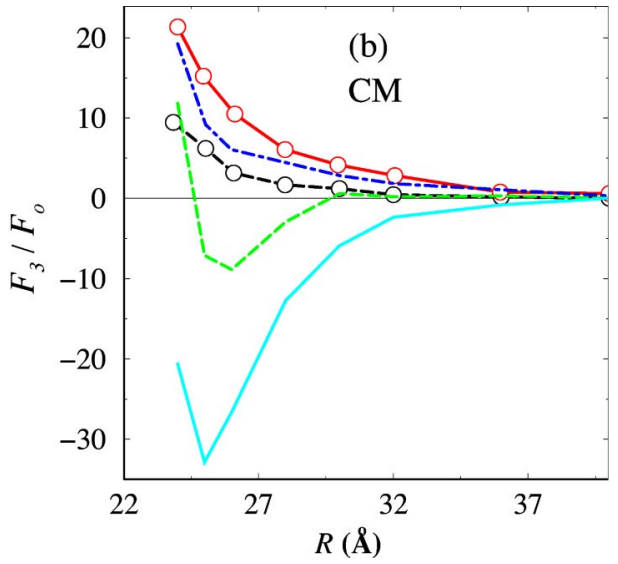

FIG. 6. (Color online) (a) Reduced electrostatic force $F_{2} / F_{0}$ and (b) entropic force $F_{3} / F_{0}$ components of the total interaction force $F(R)$ from the Fig. 5(a) for the $\mathrm{CM}$. The notation is the same as in Fig. 4. 


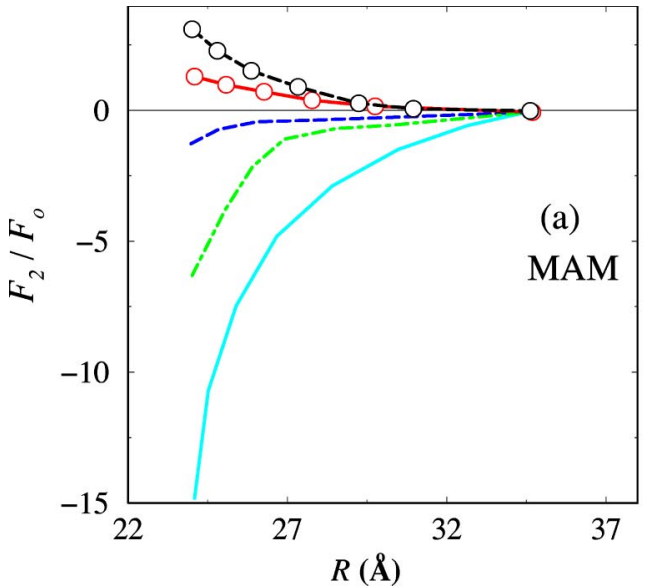

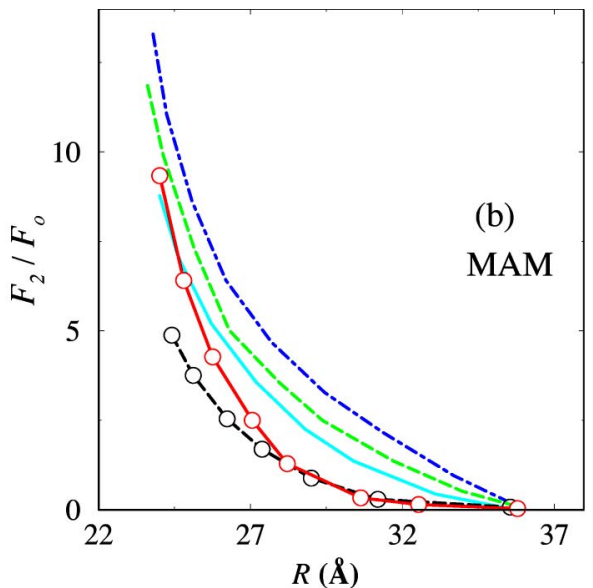

FIG. 7. (Color online) (a) Reduced electrostatic force $F_{2} / F_{0}$ and (b) entropic force $F_{3} / F_{0}$ components of the total interaction force $F(R)$ from Fig. 5(c) for the MAM. The ECM exhibits a similar trend. The notation is the same as in Fig. 4. rations and dense salt the interaction has a repulsive branch of entropic origin. However, there is a counterintuitive behavior of the force-salt dependence in the ECM and MAM: a small increase in the salt concentration $C_{s}$ suppresses the attractive interaction force $F$. As $C_{s}$ increases further, the DNA-DNA interaction force becomes strongly repulsive over a broad range of separation distances. At even higher $C_{s}$, the interaction force $F$ is completely screened out and descends toward zero in accordance with the classical double layer theories. The force-salt dependence at a fixed separation distance is shown in the inset of Fig. 5(c). We denote this trend salt-enhanced repulsion. It is in complete contrast to salt-induced destabilization or salt-induced coagulation which is typical for charged colloids [96]. As seen from Fig. 7 , it is the electrostatics that makes the total interaction attractive for the ECM and MAM at small salt densities. As $C_{s}$ is increased, the monovalent ions tend to replace the adsorbed divalent ions on the DNA surface. This replacement is energetically favorable, since the divalent ions gain more polarization energy in the bulk electrolyte. This results in the loss of the attractive electrostatic force and the weakening of the entropic force. At the final stage, when all the divalent sites on the DNA surface are occupied by monovalent ions, the strongly correlated fluid structure is destroyed and the entropic and electrostatic forces drop to zero.

\section{Trivalent counterions}

Overwhelmingly attractive DNA-DNA interaction forces for trivalent counterions and monovalent salt ions are plotted in Fig. 8. This attraction is stronger than for the divalent counterions, in accordance with the results of Ref. [61]. Similar to the divalent counterion case, the DNA-DNA attraction in the CM is much stronger than the attraction in the MAM for a given salt density. Evidently this is related to the different counterion condensation patterns for different DNA models [77]. The attraction in the CM for small added salt densities $C_{s}$ has again an entropic origin [78]. For higher salt densities, however, the attraction in the total interaction force at the short separation distances is electrostatically driven. There is no salt-enhanced repulsion for the ECM and MAM for the salt densities indicated in Fig. 8. Test runs with higher salt concentrations (not shown here) reveal that the salt- enhanced repulsion in the MAM does appear at $C_{s}$ $=1.2 \mathrm{~mole} / \mathrm{l}$ when the repulsive interaction force starts to descend towards zero.

\section{SIMULATION RESULTS FOR MULTIVALENT SALT}

\section{A. Monovalent counterions}

The results obtained in previous sections indicate that the multivalent counterions generate strong correlations inside the system and induce an electrostatic attraction between the DNA rods in the monovalent salt system. An intriguing question is how this DNA-DNA attraction relates to DNA overcharging, e.g., to the charge inversion of DNA induced by a single layer of condensed ions. Going back to the single DNA case, considered in Ref. [77], we observed no overcharging for multivalent counterions and monovalent salt ions. Thus we conclude that the overcharging effect is not a necessary condition for a DNA-DNA attraction to take place. In other words, the electrostatic ion correlations, which are not strong enough to induce the macroion overcharging, are able to induce an attractive intermolecular force. On the other hand, in Ref. [77] we have seen a DNA overcharging when multivalent salt ions were pumped into the DNA suspension. Since in the dense multivalent salt solutions the bulk charge density profiles have an oscillating behavior [97], the strongly overcharged DNA is surrounded by a sequence of radially alternating charged layers of ions. The period of these oscillations is a decreasing function of the salt density. The question we want to address here is to what extent the existence of such layers affects the DNA-DNA interaction.

The total interaction forces for the divalent salt and monovalent counterions are depicted in Fig. 9. For all three DNA models, the DNA-DNA interaction is repulsive at small salt densities $C_{s}$ and becomes attractive for a sufficiently high $C_{s}$. The DNA-DNA repulsion at lower salt is composed from the repulsive $F_{2}$ and $F_{3}$ components of the interaction force $F$. In a similar way the attraction at a dense salt, which is strong for the CM and weaker for MAM, arises from both attractive electrostatic and entropic forces. For like-charged colloid particles at similar parameters for the small ions, an attraction was reported only for the electrostatic component of the interaction force [98]. A multivalent salt-induced pre- 

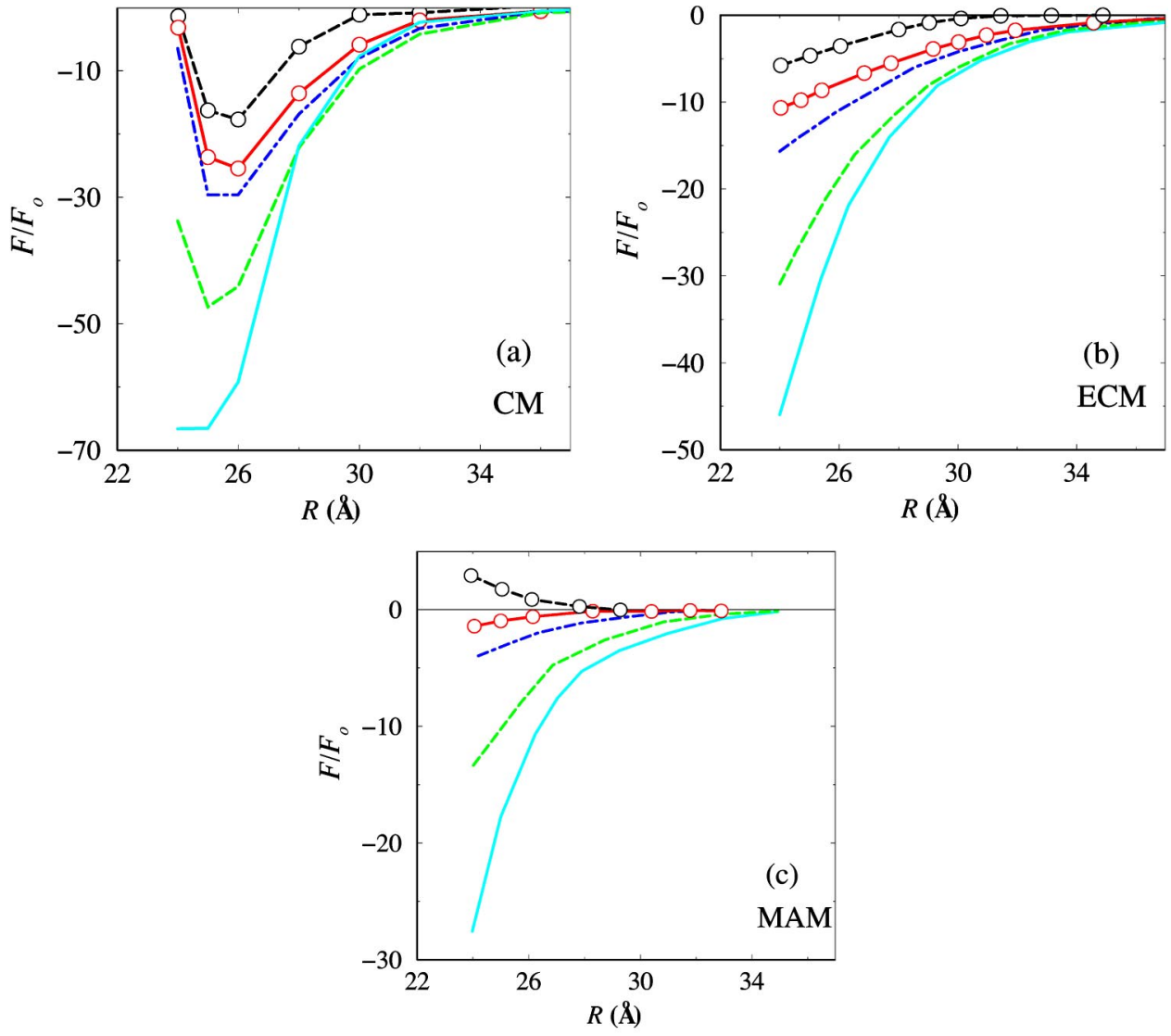

cipitation of polyelectrolyte solution is also addressed in Ref. [99].

The DNA-DNA attraction at the divalent salt density $C_{s}$ $=0.71 \mathrm{~mole} / 1$ in Fig. 9 corresponds to the overcharging of a single DNA molecule, see Ref. [77]. Thus, an overcharging and entailed charge layers near the DNA surface due to the bulk charge-density oscillations [97,100] correlate with the DNA-DNA attraction in the divalent salt [101], at least when monovalent counterions are involved. Note that the period of

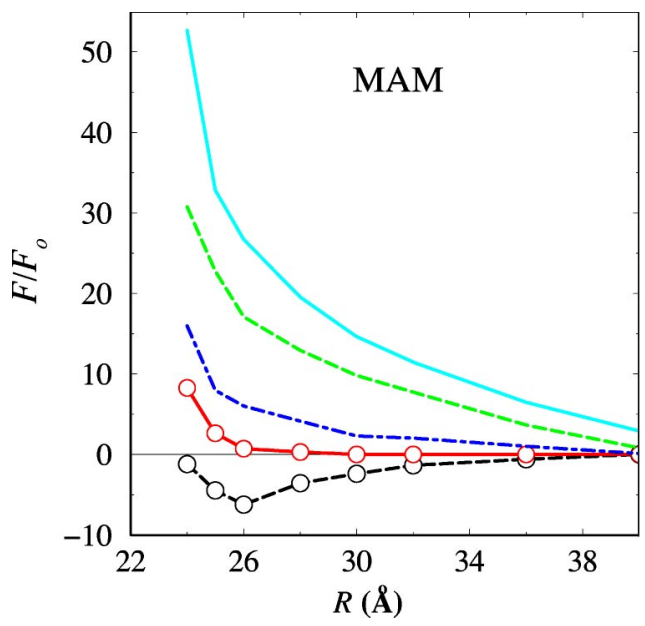

FIG. 9. (Color online) Reduced DNA-DNA interaction force $F / F_{0}$ vs separation distance $R$ for a divalent salt and monovalent counterions (parameter set 4 of Table I) for the MAM. The CM and ECM exhibit similar trends. The notation is the same as in Fig. 4. oscillations is less than the maximal surface-to-surface separation distance shown in Fig. 9.

\section{B. Trivalent counterions}

Simulation results for divalent salt and trivalent counterions are illustrated in Fig. 10. Now the DNA-DNA interaction force and both of its components $F_{2}$ and $F_{3}$ (not shown here) are strongly attractive for all the calculated salt densities. We note that at small salt densities, where no overcharging was found for a single DNA molecule [77], the obtained attractive force relates to strong charge correlations in the system [97]. Broadly speaking, there is a competition between the correlations that induce an attraction between the DNA molecules (multivalent counterion induced correlations) and the overcharging effect (multivalent salt-induced correlations). To understand the physics of this competition we have analyzed the tendencies of these two correlation effects against the increase of salt density. Figure 10 shows that the interaction forces decay monotonically with increasing salt concentration $C_{s}$. This trend is in contrast to the results for a divalent salt and monovalent counterions in Fig. 9, where more salt induces more attraction. Thus, the main contribution to the DNA-DNA attraction comes from the strong correlations between the two strongly correlated layers of trivalent counterions on the DNA surfaces. Pumping more divalent salt into the system destroys the two-dimensional crystal structure and associated correlations. However the interaction force remains attractive due to the additional overcharging of the DNA and apparent charge oscillations in bulk. As a re- 


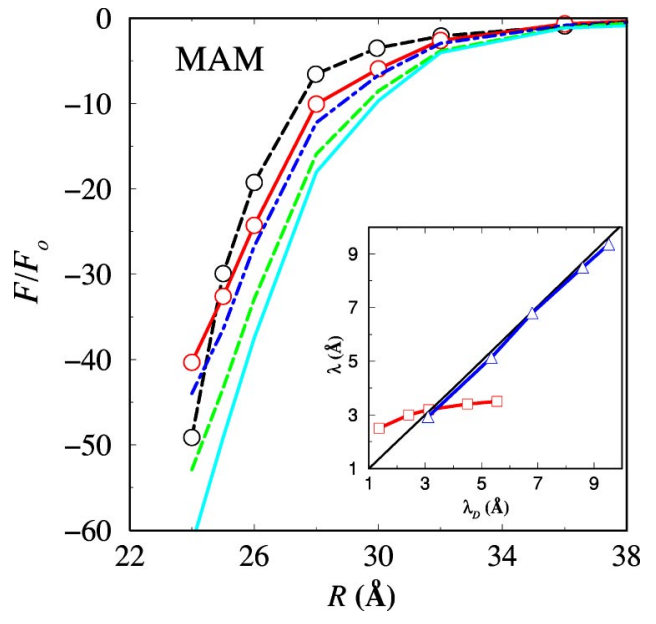

FIG. 10. (Color online) Reduced DNA-DNA interaction force $F / F_{0}$ vs separation distance $R$ for a divalent salt and trivalent counterions (parameter set 5 of Table I) and for the MAM. The CM and ECM exhibit similar trends. The notation is the same as in Fig. 4. The inset shows the relation between the decay length $\lambda$ and the Debye screening length $\lambda_{D}$ : line with triangles-for monovalent counterions and salt ions (parameter set 1 of Table I), line with squares-for current set of parameters.

sult, the DNA-DNA attraction survives for a dense salt, opposite to the case of trivalent counterions and monovalent salt shown in Fig. 8. The inset to Fig. 10 shows the relation between the Debye length $\lambda_{D}$ and the actual decay length $\lambda$ of interaction forces, which is defined through the fitting of the force curves by a function $H(r)=C \lambda K_{1}(r / \lambda) / K_{1}^{2}[D /$ $(2 \lambda)]$ over the whole range of separation distances. Here $H(x)$ is an effective interaction force between two parallel homogeneously charged cylinders, $K_{1}(x)$ is a Bessel function of imaginary argument, and $C$ is a fitting parameter which relates to the effective linear charge density of rods. It is seen that for a low salt concentrations the actual decay length in system is less than the mean-field theory based screening length $\lambda_{D}$. However for dense salt the actual decay length $\lambda$ is higher than the Debye length $\lambda_{D}$. For monovalent salt and counterions both $\lambda_{D}$ and $\lambda$ (extracted from the fitting of curves in Fig. 4) almost coincide. A comprehensive analysis of the relation between these two quantities in bulk electrolyte is given in Refs. [97,101].

\section{CONCLUSION}

We have studied the interaction forces between a pair of DNA molecules in an electrolyte that contains a mixture of monovalent and multivalent ions. Three models for the DNA shape, employed in our simulations, indicate the importance of the DNA geometry on the electrostatic and entropic forces in the DNA conformations. We show that the DNA-DNA attraction is directly related to the charge correlations in ionic solution. We distinguish between multivalent counterion and multivalent salt induced attractions. In general, the higher the mean valency of microions in the solution, the stronger is the mutual attraction between like-charged mac- roions. Below we shortly summarize the main results of this manuscript.

For the multivalent counterions, the DNA shape is an essential contributor to the interaction forces. Thus we have the following points.

(i) For no added salt the DNA-DNA attraction in the CM is related to the Coulomb depletion mechanism. This ion depletion effect results in an attractive entropic force. However such an attraction mechanism does not exist in the MAM.

(ii) For the nonzero added salt cases, an attraction in the $\mathrm{CM}$ is mainly due to a combination of electrostatic and entropic forces. However the attractive force in the MAM always has an electrostatic origin. The entropic force in the MAM is always repulsive.

(iii) There is a nonmonotonic force-salt dependence at a fixed separation distance. For divalent counterions, there is a change of the interaction force from the repulsion to an attraction, and then back to zero, which we call salt-enhanced repulsion.

For the multivalent added salt, the DNA shape has a minor effect on the interaction forces. DNA-DNA interaction forces are stronger for the CM than for the MAM. Further trends are as follows:

(i) An increase of the divalent salt density at a fixed monovalent ion number drives the DNA-DNA interaction force towards attraction. Both DNA molecules are overcharged in the attractive force regime.

(ii) For trivalent counterions the addition of divalent salt decreases the DNA-DNA attraction. The more the DNA becomes overcharged, the less is the attraction between the DNA molecules.

(iii) The correlation effects related to multivalent counterions have a greater influence on the DNA attraction than the correlation effects related to multivalent salt ions. In other words, an overcharging-induced attraction is weak compared to the counterion-induced attraction.

We would like to make some comments about the range of the DNA-DNA attraction which directly influences the phase diagram of DNA solutions [102]. Compared to the Debye screening length, the attraction forces between the DNA molecules have larger decay length at high electrolyte concentrations. For example, in Fig. 10 the range of attraction $\lambda$ for $C_{s}=0.71$ mole $/ 1$ is nearly two times larger than $\lambda_{D}$. We suppose that this effect arises directly from the DNA overcharging in highly charged ionic solutions and the bulk electrolyte oscillations. In contrast, for colloids, usually the attraction is short ranged in comparison with the screening length [9]. Thus, the calculated attractive DNA-DNA forces can lead to phase separation in DNA solutions. Unfortunately, the exact asymptotes of our calculated forces can not be obtained straightforwardly in order to compare our findings with the results of Refs. [97,101] due to the following reasons: (i) for true asymptotes one needs large separation distances; (ii) while the electrostatic forces pertain the same decay length that emerges in the charge density of electrolyte in bulk, a similar assumption for entropic forces does not apply.

Finally, let us comment on some limitations of our model. Our choice of a continuum dielectric model has the intention 
to separate the purely electrostatic effects from the effects of hydration and the molecular structure of the solvent. In many applications, including strong polyelectrolytes and high salt concentrations, continuum dielectric models (the primitive electrolyte model) have been successful. However, strictly speaking, the continuum model is not justified at small ionDNA and ion-ion separations where the molecular nature of the solvent is no longer negligible [84,103].

Other effects not accounted for in the dielectric continuum model are the dielectric discontinuity and dielectric saturation effects. The former effect emerges due to the po- larization of the DNA surface and affects the ion distribution outside the DNA core. It rapidly drops off for large distances from the surface [35]. The latter effect is related to the water anisotropy near the DNA surface and can be accounted for through a distance dependent $\epsilon$ in the electrostatic potentials $[18,75]$.

\section{ACKNOWLEDGMENTS}

E.A. thanks A. Cherstvy, R. Podgornik, and A. Parsegian for fruitful discussions of some results of the current paper.
[1] J. Pelta, F. Livolant, and J.-L. Sikorav, J. Biol. Chem. 271, 5656 (1996).

[2] V. A. Bloomfield, Biopolymers 31, 1471 (1991); 44, 269 (1997); Curr. Opin. Struct. Biol. 6, 334 (1996).

[3] X. Shui, L. McFail-lsom, G. G. Hu, and L. D. Williams, Biochemistry 37, 8341 (1998).

[4] H. A. Tajmir-Riahi, M. Naoui, and R. Ahmad, J. Biomol. Struct. Dyn. 11, 83 (1993); I. Fita, J. L. Campos, L. C. Puigjaner, and J. A. Subriana, J. Mol. Biol. 167, 157 (1983).

[5] A. M. Wierenga and A. P. Philipse, J. Colloid Interface Sci. 180, 360 (1996).

[6] R. Kjellander, S. Marčelja, and J. P. Quirk, J. Colloid Interface Sci. 126, 194 (1988).

[7] G. M. Kepler and S. Fraden, Phys. Rev. Lett. 73, 356 (1994).

[8] J. C. Crocker and D. G. Grier, Phys. Rev. Lett. 77, 1897 (1996).

[9] A. E. Larsen and D. G. Grier, Nature (London) 385, 231 (1997).

[10] H. Wennerström, A. Khan, and B. Lindman, Adv. Colloid Interface Sci. 34, 433 (1991).

[11] P. Kekicheff, S. Marčelja, T. J. Senden, and V. E. Shubin, J. Chem. Phys. 99, 6098 (1993).

[12] W. Saenger, Principles of Nucleic Acid Structure (SpringerVerlag, New York, 1984).

[13] J. A. Benbasat, Biochemistry 23, 3609 (1984).

[14] H. Deng and V. A. Bloomfield, Biophys. J. 77, 1556 (1999).

[15] F. J. Solis and M. O. de la Cruz, Phys. Rev. E 60, 4496 (1999).

[16] G. Lamm and G. R. Pack, Int. J. Quantum Chem. 20, 213 (1993); Biopolymers 34, 227 (1994).

[17] L. G. Nilsson, L. Gulbrand, and L. Nordenskiöld, Mol. Phys. 72, 177 (1991).

[18] A. P. Lyubartsev and L. Nordenskiöld, J. Phys. Chem. 99, 10373 (1995).

[19] H. Deng, V. A. Bloomfield, J. M. Benevides, and G. J. Thomas, Jr., Nucleic Acids Res. 28, 3379 (2000).

[20] M. O. Khan and B. Jönsson, Biopolymers 49, 121 (1999).

[21] B.-Y. Ha and A. J. Liu, Phys. Rev. Lett. 81, 1011 (1998).

[22] M. Deserno, A. Arnold, and Ch. Holm, Macromolecules 36, 249 (2003).

[23] L. Guldbrand, B. Jönsson, H. Wennerström, and P. Linse, J. Chem. Phys. 80, 2221 (1984); L. Guldbrand, L. G. Nilsson, and L. Nordenskiöld, ibid. 85, 6686 (1986).

[24] P. G. Bolhuis, T. Åkesson, and B. Jönsson, J. Chem. Phys. 98, 8096 (1993).
[25] X. E. Cai and J. Yang, Biophys. J. 82, 357 (2002).

[26] E. J. W. Vervey and J. T. G. Overbeek, Theory of Stability of Lyophobic Colloids (Elsevier, Amsterdam, 1948).

[27] E. S. Reiner and C. J. Radke, Adv. Colloid Interface Sci. 47, 59 (1993).

[28] P. Mills, C. F. Anderson, and M. T. Record, J. Phys. Chem. 89, 3984 (1985).

[29] R. A. Marcus, J. Chem. Phys. 23, 1057 (1955).

[30] F. Fogolari, P. Zuccato, G. Esposito, and P. Viglino, Biophys. J. 76, 1 (1999).

[31] P. J. Lin-Chung and A. K. Rajagopal, Phys. Rev. E 52, 901 (1995).

[32] M. K. Gilson, M. E. Davis, B. A. Luty, and J. A. McCammon, J. Phys. Chem. 97, 3591 (1993).

[33] K. Wagner, E. Keyes, T. W. Kephart, and G. Edwards, Biophys. J. 73, 21 (1997).

[34] V. Vlachy, Annu. Rev. Phys. Chem. 50, 145 (1999).

[35] S. Gavryushov and P. Zielenkiewicz, Biophys. J. 75, 2732 (1998).

[36] J. Rescic, V. Vlachy, L. B. Bhuiyan, and C. W. Outhwaite, J. Chem. Phys. 107, 3611 (1997).

[37] T. Das, D. Bratko, L. B. Bhuiyan, and C. W. Outhwaite, J. Chem. Phys. 99, 410 (1995).

[38] R. R. Netz and H. Orland, Eur. Phys. J. E 1, 203 (2000).

[39] A. K. Sood, Solid State Phys. 45, 1 (1991).

[40] C. J. Coen, H. W. Blanch, and J. M. Prausnitz, AIChE J. 41, 996 (1995).

[41] V. Vlachy, H. W. Blanch, and J. M. Prausnitz, AIChE J. 39, 215 (1993).

[42] M. Farnum and C. Zukoski, Biophys. J. 76, 2716 (1999).

[43] O. Spalla, Curr. Opin. Colloid Interface Sci. 5, 5 (2000).

[44] A. Weiss and M. Ballauff, Colloid Polym. Sci. 278, 1119 (2000).

[45] R. Kjellander and S. Marčelja, Chem. Phys. Lett. 49, 112 (1984); C. L. Steven and T. M. Glenn, Adv. Chem. Phys. 56, 141 (1984); S. E. Feller and D. A. McQuarrie, Mol. Phys. 80, 721 (1993).

[46] N. Grønbech-Jensen, K. M. Beardmore, and P. Pincus, Physica A 261, 74 (1998); A. W. C. Lau et al., Phys. Rev. E 63, 051604 (2001); P. Pincus and S. A. Safran, Europhys. Lett. 42, 103 (1998).

[47] L. Harnau and J.-P. Hansen, J. Chem. Phys. 116, 9051 (2002).

[48] R. Kjellander and S. Marčelja, J. Phys. Chem. 90, 1230 (1986). 
[49] P. Attard, D. J. Mitchell, and B. W. Ninham, J. Chem. Phys. 88, 4987 (1988); 89, 4358 (1988).

[50] R. Podgornik, J. Phys. A 23, 275 (1990).

[51] M. J. Stevens and M. O. Robbins, Europhys. Lett. 12, 81 (1990).

[52] A. W. C. Lau, D. Levine, and P. Pincus, Phys. Rev. Lett. 84, 4116 (2000).

[53] R. van Roij, J. Phys.: Condens. Matter 12, A263 (2000).

[54] R. van Roij and J.-P. Hansen, Phys. Rev. Lett. 79, 3082 (1997); R. van Roij, M. Dijkstra, and J.-P. Hansen, Phys. Rev. E 59, 2010 (1999).

[55] O. Spalla and L. Belloni, J. Chem. Phys. 95, 7689 (1991); L. Belloni and O. Spalla, ibid. 107, 465 (1997).

[56] B. Hribar and V. Vlachy, J. Phys. Chem. 101, 3457 (1997).

[57] R. R. Netz and H. Orland, Europhys. Lett. 45, 726 (1999); R. R. Netz, Eur. Phys. J. E 5, 557 (2001); Phys. Rev. E 60, 3174 (1999); A. G. Moreira and R. R. Netz, Phys. Rev. Lett. 87, 078301 (2001).

[58] D. Goulding and J.-P. Hansen, Europhys. Lett. 46, 407 (1999).

[59] G. Ariel and D. Andelman, Phys. Rev. E 67, 011805 (2003).

[60] Y. Burak, G. Ariel, and D. Andelman Biophys. J. 85, 2100 (2003).

[61] N. Grønbech-Jensen, R. J. Mashl, R. F. Bruinsma, and W. M. Gelbart, Phys. Rev. Lett. 78, 2477 (1997); N. GrønbechJensen and K. M. Beardmore, Physica A 261, 74 (1998).

[62] Y. Levin, J. J. Arenzon, and J. F. Stilck, Phys. Rev. Lett. 83, 2680 (1999); J. J. Arenzon, J. F. Stilck, and Y. Levin, Eur. Phys. J. B 12, 79 (1999).

[63] J. J. Delrow, J. A. Gebe, and J. M. Schurr, Biopolymers 42, 455 (1997).

[64] P. Linse and V. Lobaskin, Phys. Rev. Lett. 83, 4208 (1999); J. Chem. Phys. 112, 3917 (2000).

[65] R. Kjellander, T. Akesson, B. Jönsson, and S. Marčelja, J. Chem. Phys. 97, 1424 (1992).

[66] I. Rouzina and V. A. Bloomfield, J. Chem. Phys. 100, 9977 (1996).

[67] D. B. Lukatsky and S. A. Safran, Phys. Rev. E 60, 5848 (1999).

[68] C. S. Murthy, R. J. Bacquet, and P. J. Rossky, J. Phys. Chem. 89, 701 (1985).

[69] V. Vlachy and A. D. J. Haymet, J. Chem. Phys. 84, 5874 (1986).

[70] M. D. Paulsen, C. F. Anderson, and M. T. Record, Biopolymers 27, 1249 (1988).

[71] P. Mills, M. D. Paulsen, C. F. Anderson, and M. T. Record, Jr., Chem. Phys. Lett. 129, 155 (1986).

[72] M. D. Paulsen, B. Richey, C. F. Anderson, and M. T. Record, Jr., Chem. Phys. Lett. 139, 448 (1987).

[73] A. Katchalsky, Pure Appl. Chem. 26, 327 (1971).

[74] B. Jayaram and D. L. Beveridge, Annu. Rev. Biophys. Biomol. Struct. 25, 367 (1996).

[75] L. Yang, A. Weerasinghe, and B. M. Pettitt, Biophys. J. 69, 1519 (1995).

[76] E. Allahyarov and H. Löwen, Phys. Rev. E 62, 5542 (2000).

[77] E. Allahyarov, H. Löwen, and G. Gompper, Phys. Rev. E 68, 061903 (2003).

[78] E. Allahyarov, I. D’Amico, and H. Löwen, Phys. Rev. Lett. 81, 1334 (1998).

[79] D. C. Rau, B. Lee, and V. A. Parsegian, Proc. Natl. Acad. Sci. U.S.A. 81, 2621 (1984); R. Podgornik, D. C. Rau, and V. A.
Parsegian, Biophys. J. 66, 962 (1994); Macromolecules 22, 1780 (1989); H. H. Strey, V. A. Parsegian, and R. Podgornik, Phys. Rev. Lett. 78, 895 (1997); R. Podgornik, H. H. Strey, K. Gawrisch, D. C. Rau, A. Rupprecht, and V. A. Parsegian, Proc. Natl. Acad. Sci. U.S.A. 93, 4261 (1996); S. Leikin, V. A. Parsegian, D. C. Rau, and R. P. Rand, Annu. Rev. Phys. Chem. 44, 369 (1993).

[80] J. L. Barrat and J. F. Joanny, Adv. Chem. Phys. 94, 1 (1996); V. A. Bloomfield and I. Rouzina, Methods Enzymol. 295, 364 (1999); J. L. Hecht, B. Honig, Y. K. Shin, and W. L. Hubbell, J. Phys. Chem. 99, 7782 (1995).

[81] A. A. Kornyshev and S. Leikin, J. Chem. Phys. 107, 3656 (1997); Proc. Natl. Acad. Sci. U.S.A. 95, 13579 (1998); Biophys. J. 75, 2513 (1998); J. Chem. Phys. 108, 7035(E) (1998); A. G. Cherstvy, A. A. Kornyshev, and S. Leikin, J. Phys. Chem. B 106, 13362 (2002).

[82] A. P. Lyubartsev and L. Nordenskiöld, J. Phys. Chem. B 101, 4335 (1997).

[83] J. C. G. Montoro and J. L. F. Abascal, J. Chem. Phys. 103, 8273 (1995); 109, 6200 (1998); J. L. F. Abascal and J. C. G. Montoro, ibid. 114, 4277 (2001).

[84] L. E. Guldbrand, T. R. Forester, and R. M. Lynden-Bell, Mol. Phys. 67, 473 (1989); B. Jayaram, K. Sharp, and B. Honig, Biopolymers 28, 975 (1989); J. Conrad, M. Troll, and B. H. Zimm, ibid. 27, 1711 (1988); D. Hochberg, T. W. Kephart, and G. Edwards, Phys. Rev. E 49, 851 (1994); D. Hochberg, G. Edwards, and T. W. Kephart, ibid. 55, 3765 (1997); G. Edwards, D. Hochberg, and T. W. Kephart, ibid. 50, R698 (1994).

[85] S. S. Zakharova, S. U. Egelhaaf, L. B. Bhuiyan, D. Bratko, and J. R. C. van der Maarel, J. Chem. Phys. 111, 10706 (1999).

[86] D. B. Lukatsky, S. A. Safran, A. W. C. Lau, and P. Pincus, Europhys. Lett. 58, 785 (2002); R. Kjellander, A. P. Lyubartsev, and S. Marčella, J. Chem. Phys. 114, 9565 (2001); R. Messina, C. Holm, and K. Kremer, Phys. Rev. E 64, 021405 (2001); Eur. Phys. J. E 4, 363 (2001); Phys. Rev. Lett. 85, 872 (2000).

[87] M. Feig and B. M. Pettitt, Biophys. J. 77, 1769 (1999).

[88] J. Lekner, Physica A 176, 485 (1991); Mol. Simul. 20, 357 (1998)

[89] B. S. Fujimoto, J. M. Miller, N. S. Ribeiro, and J. M. Schurr, Biophys. J. 67, 304 (1994).

[90] M. P. Allen and D. J. Tildesley, Computer Simulation of Liquids (Oxford University Press, Oxford, 1987).

[91] N. Korolev, A. P. Lyubartsev, A. Rupprecht, and L. Nordenskiöld, Biophys. J. 77, 2736 (1999); N. Korolev, A. P. Lyubartsev, L. Nordenskiöld, and A. Laaksonen, J. Mol. Biol. 38, 907 (2001).

[92] A. Papadopoulou, E. D. Becker, M. Lupkowski, and F. van Swol, J. Chem. Phys. 98, 4897 (1993).

[93] P. Attard, J. Chem. Phys. 107, 3230 (1997), and references therein.

[94] B. R. Svennson and C. E. Woodward, Mol. Phys. 64, 247 (1988).

[95] This effect resembles the so called "hydration attraction" [see D. C. Rau and V. A. Parsegian, Biophys. J. 61, 260 (1992)], when the entropic "push" from the outside area is generated by the water particles. A similar effect, called "a counterion evaporation" [see C. Fleck and H. H. von Grünberg, Biophys. J. 63, 061804 (2001)] exists between the oppositely charged rod and surface. 
[96] E. Allahyarov, H. Löwen, J.-P. Hansen, and A. A. Louis, Phys. Rev. E 67, 051404 (2003); Y. U. Moon, C. O. Anderson, H. W. Blanch, and J. M. Prausnitz, Fluid Phase Equilib. 168, 229 (2000).

[97] P. Attard, Phys. Rev. E 48, 3604 (1993).

[98] J. Z. Wu, D. Bratko, H. W. Blanch, and J. M. Prausnitz, Proc. Natl. Acad. Sci. U.S.A. 95, 15169 (1998).

[99] M. Olvera de la Cruz, L. Belloni, M. Delsanti, J. P. Dalbiez, O. Spalla, and M. Drifford, J. Chem. Phys. 103, 5781 (1995).

[100] M. Deserno, F. Jimenez-Angeles, C. Holm, and M. LozadaCassou, J. Phys. Chem. B 105, 10983 (2001). See also L.
Degreve, M. Lozada-Cassou, E. Sanchez, and E. GonzalezTovar, J. Chem. Phys. 98, 8905 (1993); M. Lozada-Cassou, J. Phys. Chem. 87, 3729 (1983).

[101] The effect of charge inversion and electrolyte layering on the mean electrostatic potential of plane is addressed in P. Attard, Adv. Chem. Phys. 92, 1 (1996); J. Phys. Chem. 99, 14174 (1995).

[102] H. N. W. Lekkerkerker, W. C. K. Poon, P. N. Pusey, A. Stroobants, and P. B. Warren, Europhys. Lett. 20, 559 (1992).

[103] E. Allahyarov and H. Löwen, Phys. Rev. E 63, 041403 (2001). 